\title{
Uso de nanomateriales magnéticos para la remoción de arsénico del agua para consumo humano*
}

\author{
Barrientos J.E., ${ }^{1}$ Matutes A. J. ${ }^{2}$
}

Resumen: Se obtuvieron nanomateriales magnéticos por medios físicos como lo es el aerosol asistido por deposición de vapor (ACCVD) y medios químicos (coprecipitación química), los cuales demostraron tener una excelente capacidad de remoción de arsénico (As) y otras impurezas contenidas en agua empleada para consumo humano, esta opción de tratamiento de agua contaminada con As es una alternativa viable al ser comparada con otros métodos comerciales de tratamiento disponibles en el mercado. Los materiales obtenidos por dos técnicas de síntesis se analizaron desde el punto de vista técnico y económico; de acuerdo con su costo de obtención y la capacidad de remoción; en ambos procesos de síntesis se usaron reactivos de alta pureza: Cloruro Férrico $\left(\mathrm{FeCl}_{3} \cdot 6 \mathrm{H}_{2} \mathrm{O},\left(\mathrm{JT}\right.\right.$ Baker)), Cloruro Ferroso $\left(\mathrm{FeCl}_{2} \cdot 4 \mathrm{H}_{2} \mathrm{O}\right.$, (JT Baker)), agua tridestilada (JT Baker), e Hidróxido de Amonio ( $\mathrm{NH} 4 \mathrm{OH})$. El tamaño y composición de las nanopartículas fueron conseguidos variando las condiciones experimentales, se obtuvo Fierro $(\mathrm{Fe})$ metálico y Hematita $\left(\mathrm{Fe}_{2} \mathrm{O}_{3}\right)$ por métodos físicos, donde la capacidad de remoción es del 100\% a los 5 minutos de contacto con el agua contaminada de arsénico; también se obtuvo magnetita por medios químicos que al mismo tiempo de contacto tuvo el $95 \%$ de remoción de arsénico. Ambos materiales se analizaron por medio de microscopia electrónica de transmisión (TEM) y microscopia electrónica de barrido (SEM). La magnetita demostró que tiene la capacidad de remover otros elementos químicos presentes en el agua para consumo humano como: $\mathrm{Cl}$, Ca, Na y $\mathrm{S}$.

PALABRAS CLAVE: nanopartículas, arsénico, ACCVD y coprecipitación.

ABSTRACT: Nanomagnetic materials were obtained by physical method like chemical vapor deposition assisted by aerosol (ACCVD) and chemical method (coprecipitacion), which materials shows high capacity to remove arsenic (As) and other content impurities in water to human consume and could be a viable alternative to remove impurities if is compared with other commercial methods available. Both materials obtaining by different methods were evaluated in obtaining cost and removal capacity technical, the reactants agents were high purity: utilizing reagent grade materials: Iron (III) Chloride Hexahydrate $\left(\mathrm{FeCl}_{3} \cdot 6 \mathrm{H}_{2} \mathrm{O}\right.$ (JT Baker)), Iron (II) Chloride Tetrahydrate $\left(\mathrm{FeCl}_{2} \cdot 4 \mathrm{H}_{2} \mathrm{O}\right.$ (JT Baker)), $\mathrm{NH}_{4} \mathrm{OH}$ and tridistilled water. Size and composition of nanoparticles were reached varying experimental conditions, metallic pure iron $(\mathrm{Fe})$ and hematite $\left(\mathrm{Fe}_{2} \mathrm{O}_{3}\right)$ were obtained by physical method. The magnetic materials removed $100 \%$ of arsenic contained in water after 5 minutes contact with water contaminated with As, also magnetite was obtained by chemical method and to 5 minutes of contact the remotion was $95 \%$. Both materials were analyzed by transmission electronic microscopy (TEM) and scanning electronic microscopy (SEM). The magnetite shows capability to remove other chemical elements presents in water consume human how: $\mathrm{Cl}, \mathrm{Ca}$, $\mathrm{Na}$ and $\mathrm{S}$.

KEY wORDS: nanoparticles, arsenic, ACCVD y coprecipitation.

\footnotetext{
* Avance de investigación.

1 Estudiante de doctorado. Instituto Nacional de Investigaciones Forestales Agrícolas y Pecuarias (INIFAP), Campo Experimental Campana-Madera. Km. 33.3 Carretera Chihuahua-Ojinaga, Aldama, Chihuahua.

Área de especialización: materiales magnéticos nanoestructurados empleados para mitigación del impacto ambiental en el sector agropecuario y forestal. Tel. 6144510601. (barrientos.eutiquio@inifap.gob.mx).

2 Ph. D. Centro de Investigación en Materiales Avanzados, S. C.

Miguel de Cervantes No. 120 CP 31109, Complejo Industrial Chihuahua, Chihuahua, México. Tel. 6144391100. Área de especialización: materiales magnéticos.
} 


\section{ANTECEDENTES}

El arsénico (As) es un elemento químico tóxico para humanos, plantas y animales. La presencia de niveles de arsénico mayores a 10 partes por billón (ppb) puede presentar problemas tales como cambios de color en la piel, malformaciones parecidas a costras en las palmas de las manos y las plantas de los pies, cáncer de piel, vejiga, riñón y pulmón, así como enfermedades presentes en los vasos sanguíneos de las piernas y los pies, presión arterial alta y trastornos de la reproducción según reporta la organización mundial de la salud en su sitio de internet. Agua empleada para consumo humano contaminada con arsénico es uno de los problemas graves alrededor del mundo [1]. Las partículas magnéticas son de gran interés para fluidos magnéticos, biotecnología, biomedicina, resonancia magnética, almacenamiento de datos y remediación ambiental. Dependiendo del estado de oxidación $\left(\mathrm{Fe}^{+2}\right.$ o $\left.\mathrm{Fe}^{+3}\right)$, los óxidos de hierro exhiben diferentes estructuras cristalinas que incluyen la hematita (á$\left.\mathrm{Fe}_{2} \mathrm{O}_{3}\right)$ y maghemita $\left(\gamma-\mathrm{Fe}_{2} \mathrm{O}_{3}\right)$, la hematita es termodinámicamente más estable que la magnetita $\mathrm{Fe}_{3} \mathrm{O}_{4}$. Hay varios métodos para producir $\mathrm{Fe}_{2} \mathrm{O}_{3}$ y $\mathrm{Fe}_{3} \mathrm{O}_{4}$, éstos incluyen rutas químicas húmedas como la coprecipitación, decomposición térmica en líquidos y microemulsiones o síntesis hidrotérmica, pero también formación de partículas en fase gaseosa en hornos tubulares o por descomposión de flama. Diferentes hornos existentes han sido usados para la síntesis de $\mathrm{Fe}_{2} \mathrm{O}_{3}$ incluyendo difusión de flamas o spray pyrolysis operando con precursores de Fe disueltos en una solución líquida [2].

\section{INTRODUCCIÓN}

Arsénico contenido en agua para consumo humano fue removido con nanomateriales magnéticos obtenidos por dos diferentes técnicas de síntesis, el tamaño promedio de las nanopartículas magnéticas obtenidas por coprecipitación química fue de $7 \mathrm{~nm}$ [3], también se sintetizaron nanopartículas magnéticas por medio de la técnica ACCVD [4] logrando tener partículas de entre 100-1000 nm de una sola fase magnética, las cuales presentan una alta capacidad de remoción de arsénico y otros elementos químicos presentes en el agua.

ACCVD es una técnica para obtener nanopartículas que muestran la posibilidad de obtener bajo una atmósfera y temperatura controlada nanopartículas magnéticas que pueden ser sintetizadas, Fe2O3 and Fe304 [5]. El proceso de generar spray es comúnmente usado para una amplia variedad de materiales en forma de polvo. El método de spray proporciona grandes ventajas sobre las técnicas de procesamiento convencional de materiales $[6,7]$.

En este trabajo, las nanopartículas magnéticas fueron obtenidas por coprecipitación química debido a que por este método se obtiene un tamaño de nanopartículas muy pequeño [8]. Se demuestra la eficiencia de remoción del arsénico contenido en agua para consumo humano por medio de las nanopartículas magnéticas y que se puede llevar a cabo un proceso sencillo y de fácil aplicación. 


\section{Procedimiento EXPERIMENTAL}

Nanopartículas magnéticas fueron sintetizadas por el método ACCVD usando materiales de grado reactivo: cloruro de hierro hexahidratado (III) (FeCl3·6H2O (JT Baker)), agua tridestilada como solvente. En la figura 1, se muestra el procedimiento utilizado para la síntesis. Donde (1) es un controlador de flujo y donde se realiza la mezcla aire-argón como gas de arrastre, (2) es un ultrasonificador que genera gotas de la solución, (3) solución inicial, (4) tubo de cuarzo de 9mm de diámetro donde pasan las gotas de la solución precursora, (5) horno y (6) es el impactor donde las nanopartículas son colectadas para su posterior análisis.

FIGURA 1. Procedimiento de síntesis de nanopartículas de hematita por ACCVD.

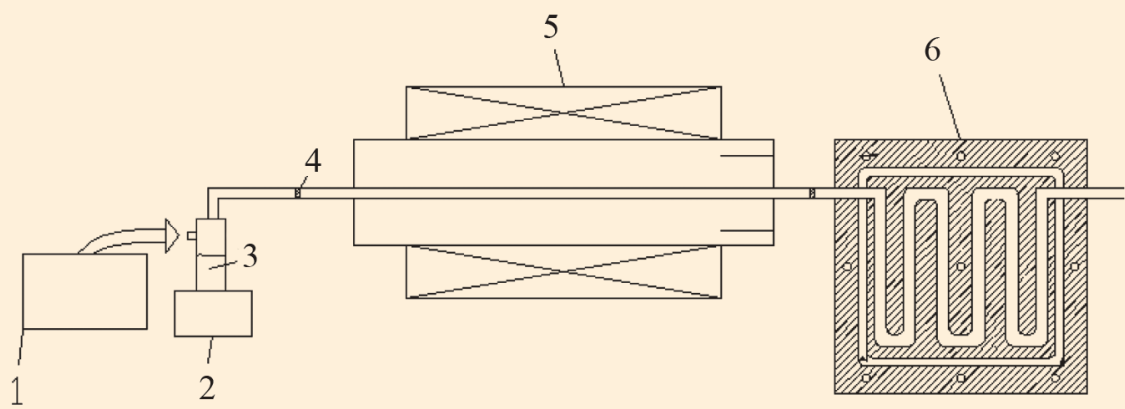

Variando la concentración de la solución de cloruro férrico $\left(\mathrm{FeCl}_{3} \cdot 6 \mathrm{H}_{2} \mathrm{O}\right)$ de 0.01 $\mathrm{M}$ a $0.1 \mathrm{M}$ y la temperatura del horno, nanopartículas magnéticas con diferentes fases fueron sintetizadas. La solución fue atomizada usando una mezcla de aire-argón como gas de arrastre, la temperatura del horno fue de $440{ }^{\circ} \mathrm{C}$ a $590^{\circ} \mathrm{C}$, donde la solución fue evaporada para la formación de nanopartículas; y se modificó la composición del gas de arrastre, la concentración de la solución así como la temperatura del horno para que el tamaño y composición de las nanopartículas pudiera ser modificado. En la tabla 1, se puede observar la variación de la concentración de la solución, la relación aire-argón que hace posible obtener Fe o hematita, así como obtener partículas de diferentes tamaños. La hematita es simple de obtener por este método y Fe puede ser obtenido por la ausencia de oxígeno.

La síntesis de materiales magnéticos por medio del método de la coprecipitación química, presentada en el diagrama de flujo en la figura 1, ayuda a obtener materiales con especificaciones especiales. Dicho método consiste en mezclar una solución de cloruro férrico $\left(\mathrm{FeCl}_{3} \cdot 6 \mathrm{H}_{2} \mathrm{O}\right)$ y cloruro ferroso $\left(\mathrm{FeCl}_{2} \cdot 4 \mathrm{H}_{2} \mathrm{O}\right)$ agitándola mecánicamente hasta alcanzar una temperatura entre $60{ }^{\circ} \mathrm{C}$ y $70^{\circ} \mathrm{C}$ y se le agrega un agente precipitante como el hidróxido de amonio $\left(\mathrm{NH}_{4} \mathrm{OH}\right)$ al $10 \%$ en volumen para obtener las nanopartículas magnéticas. En este método de obtención de nanopartículas, al controlar el pH y la temperatura de la solución, se controla el tamaño y la fase deseada del material magnético. Después de la coprecipitación química, se lavan las nanopartículas con agua destilada para eliminar los residuos no deseados y que puedan afec- 
tar el proceso de captura de arsénico y otros elementos químicos contenidos en agua contaminada.

\section{RESULTADOS Y DISCUSIÓN}

\section{Microscopia electrónica de transmisión}

En la figura 2a) se muestran anillos de un patrón de difracción característicos de magnetita y en la figura 2b) un aglomerado de partículas nanométricas, las nanopartículas tienen un tamaño promedio aproximado de $7 \mathrm{~nm}$. La composición química de las nanopartículas afecta la estructura interna de las mismas, por lo que los patrones son característicos para cada fase de los materiales magnéticos.

FIGURA 2a) y b). Nanopartículas y patrón de difracción de magnetita obtenidas por medio químico.
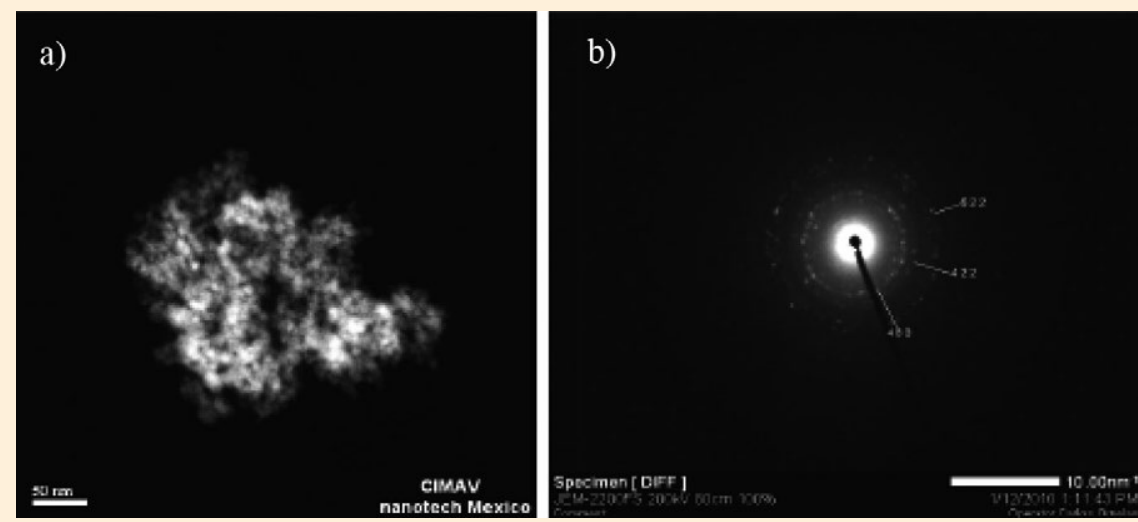

Fierro puro (Fe) y hematita fueron obtenidos por ACCVD; la figura 3a) muestra anillos de un patrón de difracción característicos de Fe y en la figura 3 b) se muestra una partícula con partículas nanométricas aglomeradas, las nanopartículas aglomeradas tienen un tamaño aproximado de $5 \mathrm{~nm}$. La variación en la composición fue obtenida variando la relación de aire-argón del gas de arrastre. La composición de las nanopartículas afecta la estructura interna de las partículas.

La figura 4 muestra la imagen del análisis realizado por microscopia de nanopartículas de magnetita y se puede observar la capacidad de remover otros elementos químicos presentes en al agua tomada para realizar las pruebas de remoción.

\section{MICROSCOPIA ELECTRÓNICA DE BARRIDO}

En las figuras 5a) y 5b) se muestran imágenes obtenidas por microscopia electrónica de barrido de las nanopartículas magnéticas, donde se observa que son de tamaño diferente, el tamaño de las nanopartículas obtenidas por medio químico son de un 
FIGURA 3a) y b). Nanopartículas y patrón de difracción de hematita obtenidas por medio físico.

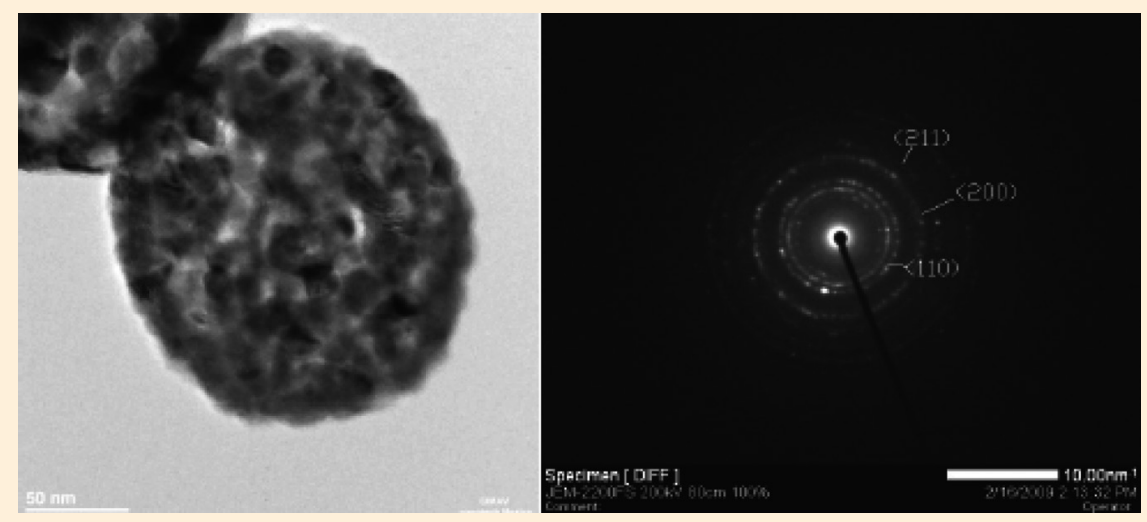

FIGURA 4. Zonas donde se realizó el análisis mediante microscopia electrónica de transmisión.

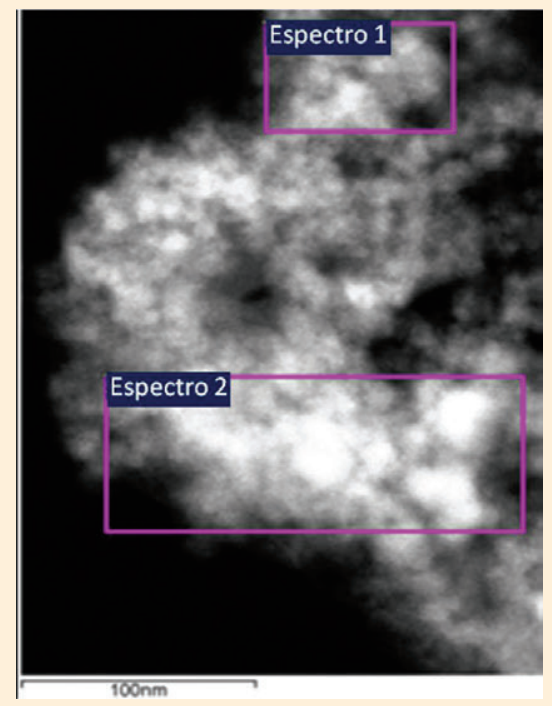

tamaño menor que las obtenidas por el medio físico, esto tiene su explicación por el proceso de nucleación y crecimiento en la obtención. Es notable la diferencia de tamaño promedio, en la figura 5a) se puede observar que el tamaño promedio está en $7 \mathrm{~nm}$ mientras que en la figura 5b) se observa un tamaño promedio de $250 \mathrm{~nm}$.

\section{Difracción de Rayos $X$}

La figura 6a) muestra un patrón característico de nanopartículas magnéticas de hematita, estás partículas fueron usadas para remover arsénico de agua contaminada, el ancho y la alta intensidad de los picos es debida al tamaño de las partículas. El tama- 
FIGURA 5a). Imagen de nanopartículas de magnetita obtenida por medio químico.

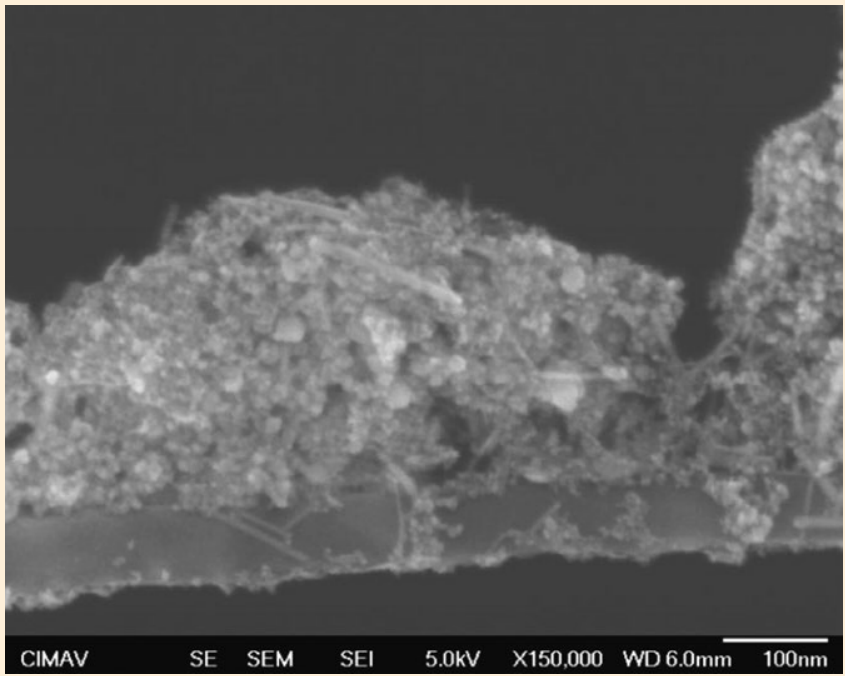

FIGURA 5b). Imagen de nanopartículas de hematita obtenidas por medio físico.

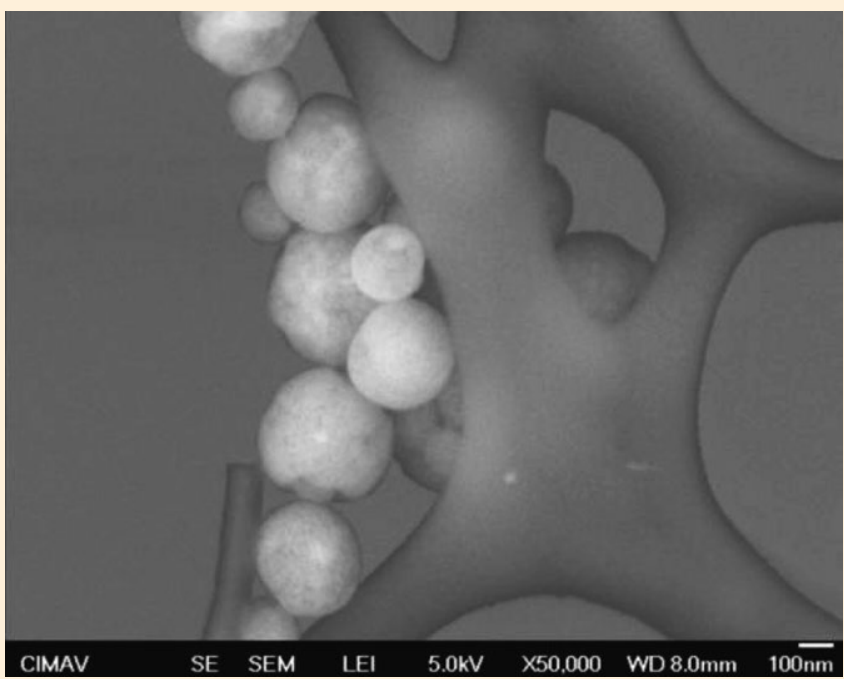

ño de las nanopartículas con una concentración de 0.01 M es de $250 \mathrm{~nm}$. La figura 6b) muestra un patrón para las nanopartículas de magnetita el tamaño promedio de las nanopartículas se puede evidenciar por ensanchamiento de los picos. Para la hematita se pueden observar picos más intensos, esto es debido a que hay presente una mayor cantidad de cristales orientados en la misma dirección en un tamaño más grande de partícula, por lo tanto al tener menor cantidad de cristales, las nanopartículas de magnetita tienen picos con menor intensidad. 
FIGURA 6a). XRD de hematita obtenida por medios físicos.

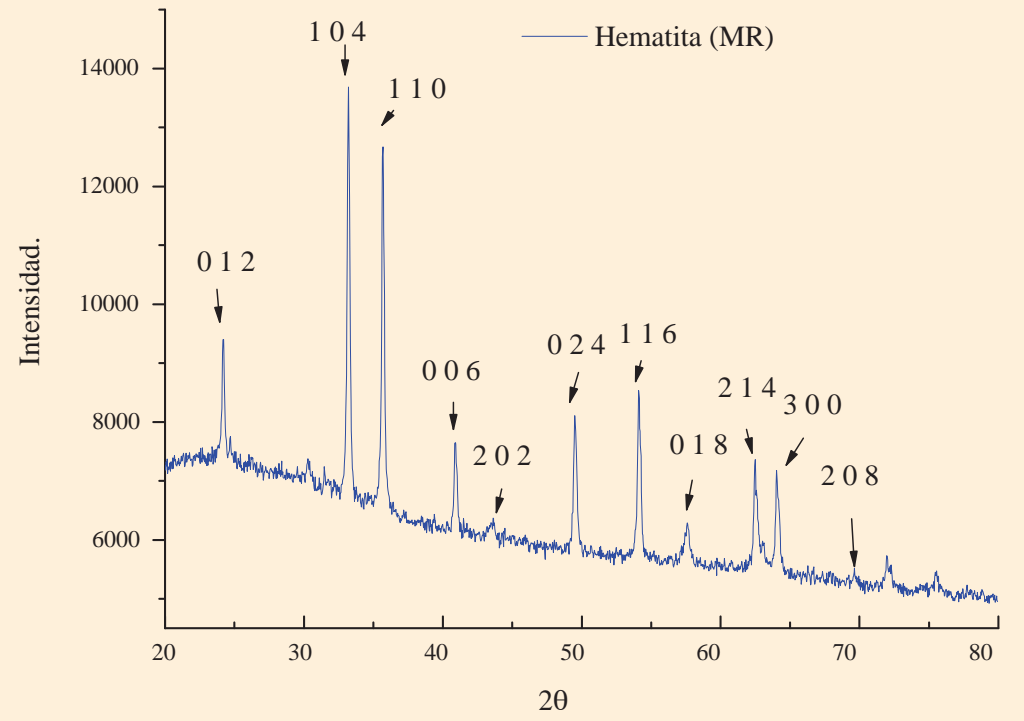

FIGURA 6b). XRD de magnetita obtenida por medios químicos.

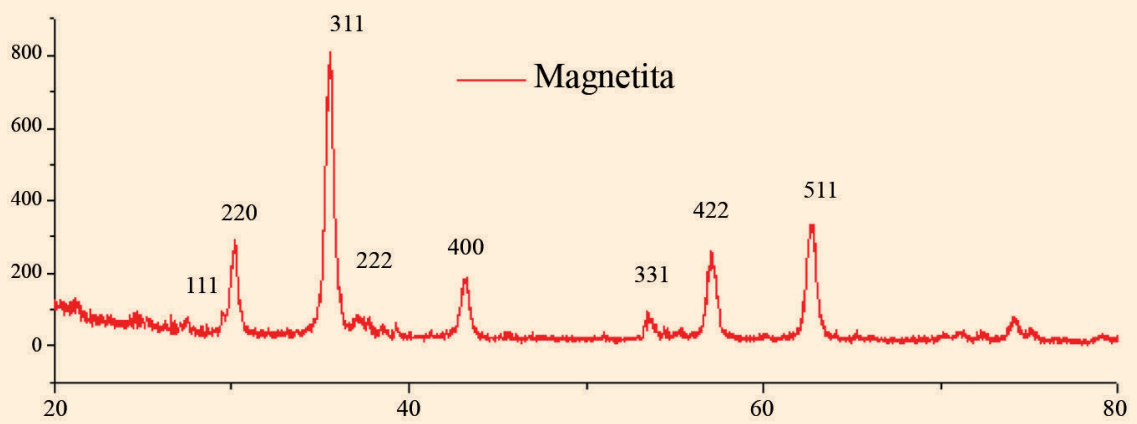

En la tabla 1 se pueden observar los elementos presentes en las nanopartículas empleadas para la remoción de arsénico contenido en agua para consumo humano, por lo que demuestran una alta capacidad de remoción de diferentes contaminantes presentes en el agua. 
TABLA 1. Elementos químicos removidos por las nanopartículas de magnetita

\begin{tabular}{|c|c|c|c|c|c|c|c|c|c|c|}
\hline $\begin{array}{c}\text { Espectros de la } \\
\text { figura 6 }\end{array}$ & $\begin{array}{c}\text { \% en } \\
\text { peso }\end{array}$ & $\mathbf{O}$ & $\mathbf{N a}$ & $\mathbf{S i}$ & $\mathbf{S}$ & $\mathbf{C 1}$ & $\mathbf{C a}$ & $\mathbf{F e}$ & As & Total \\
\hline Espectro 1 & & 42.12 & 6.69 & 9.39 & & & & 41.02 & $0.78 \pm .50$ & 100.00 \\
\hline Espectro 2 & & 38.70 & 8.05 & 6.75 & 4.76 & 2.42 & 5.63 & 33.69 & 0.00 & 100.00 \\
\hline
\end{tabular}

En la tabla 2 podemos ver el tiempo de remoción, así como el costo estimado por $\mathrm{m}^{3}$ de agua tratada con las nanopartículas por ambos métodos y la capacidad de remoción, las nanopartículas de hematita obtenidas por medio físico son más eficientes al remover al mismo tiempo de contacto que con las nanopartículas obtenidas por medio químico, pero el tiempo de obtención y la cantidad obtenida por experimento es mayor. La magnetita tiene un tiempo de obtención sumamente bajo por lo que se compensa con un porcentaje bajo de remoción comparado con la hematita.

TABLA 2. Tratamiento de agua contaminada con $0.020 \mathrm{mg} / \mathrm{L}$ de As

\begin{tabular}{|l|c|c|c|c|c|}
\hline \multicolumn{1}{|c|}{ Tipo de muestra } & As $\mathbf{m g} / \mathbf{L}$ & Fe $\mathbf{~ m g / L}$ & $\begin{array}{c}\text { Tiempo de } \\
\text { ultrasonificación (min) }\end{array}$ & $\begin{array}{c}\text { \% de } \\
\text { Remoción }\end{array}$ & $\begin{array}{c}\text { Costo de } \\
\text { remoción por } \mathbf{~ m}^{3}\end{array}$ \\
\hline $\begin{array}{l}\text { Agua contaminada } \\
\text { con arsénico. }\end{array}$ & 0.020 & 0 & 0 & 0 & 100 \\
\hline $\begin{array}{l}\text { Agua después de } \\
\text { ser tratada con } \\
\text { nanopartículas de } \\
\text { hematita. }\end{array}$ & N.D. & 0 & 5 & $\$ 400.00$ M.N. \\
\hline $\begin{array}{l}\text { Agua después de } \\
\text { ser tratada con } \\
\text { nanopartículas de } \\
\text { magnetita }\end{array}$ & 0.001 & 0 & 5 & 95 & $\$ 40.00$ M.N. \\
\hline
\end{tabular}

\section{Conclusiones}

- El tamaño de las partículas de hematita es mayor que las partículas de magnetita.

- La capacidad de remoción de las nanopartículas de hematita es del 100\% a los 5 minutos de contacto con agua contaminada y para las nanopartículas de magnetita es del 95\% para el mismo tiempo.

- La temperatura de obtención de las partículas de magnetita es de $70{ }^{\circ} \mathrm{C}$ y para la hematita es de $590^{\circ} \mathrm{C}$.

- El costo de remoción de arsénico por cada $\mathrm{m}^{3}$ es de $\$ 40.00$ pesos con nanopartículas de magnetita y de $\$ 400.00$ con nanopartículas de hematita.

\section{Referencias}

[1] J.T. Mayoa, C. Yavuza, S. Yeanb, L. Congb, H. Shipleyb, W. Yua, J. Falknera, A. Kanb, M. Tomsonb, V.L. Colvina. "The effect of nanocrystalline magnetite size on arsenic removal". Science and Technology of Advanced Materials 8 (2007) 71-75. 
[2] R. Strobel, S.E. Pratsinis. "Direct synthesis of maghemite, magnetite and wurstite nanoparticles by flame spray pyrolysis". Advanced Powder Technology 20 (2009) 190-194.

[3] Barrientos E., Matutes J., Miki M., Santillán R. Método para remover arsénico de agua para consumo humano por medio de nanopartículas magnéticas, obtenidas por coprecipitación química. Patente No.MX/a/2011/1005703.

[4] Monárrez B., Amézaga P., Barrientos E., Miki M. Proceso para sintetizar nanopartículas mesoporosas y huecas de alta área superficial. Patente No. MX/a/2012/004874.

[5] Reto Strobel, Sotiris E. Pratsinis. "Direct synthesis of maghemite, magnetite and wustite nanoparticles by flame spray pyrolysis". Advanced Powder Technology 20 (2009) 190-194.

[6] A. Gurav, T. Kodas, T. Pluym, Y. Xiong. "Aerosol processing of materials". Aerosol Sci. Technol. 19 (1993) 411-452.

[7] S.E. Pratsinis, S. Vemury. "Particle formation in gases". Powder Technol. 88(1996) 267273.

[8] T. Yavuz, Arjun Prakash, J.T. Mayo, Vicki L. Colvin. "Magnetic separation: From steel plants to biotechnology". Chemical Engineering Science 64 (2009) 2510-2521.

[9] O. Ayala-Valenzuela, J. Matutes-Aquino, R. Betancourt-Galindo, L.A. García-Cerda, O. Rodríguez Fernández, P.C. Fannin, A.T. Giannitsis. "Magnetite-cobalt ferrite nanoparticles for kerosene-based magnetic fluids". Journal of Magnetism and Magnetic Materials, vol. 294, núm. 2, julio 2005: e37-e41. 